bureau: Roche, Chugai, BMS, Abbvie, Servier, Pfizer, MSD, UCB, ESAOTE, Genévrier, Janssen, Novartis, Lilly, Biogen, Amge, Denis Jullien Consultant for: Abbott, Celgene, Eli Lilly, Janssen, MSD, Novartis, Pfizer, Serono UCB, Fresenius-Kabi, Biogen, Leo-Pharma and Sandoz, Speakers bureau: Abbott, Celgene, Eli Lilly, Janssen, MSD, Novartis, Pfizer, LeoPharma, Serono, Thao Pham Speakers bureau: Lilly, Novartis, Rene-Marc Flipo Consultant for: Advisory board: Bristol-Myers Squibb DOI: 10.1136/annrheumdis-2019-eular.2573

\section{AB0704 1 EFFECTIVENESS AND SAFETY OF INFLIXIMAB AND GOLIMUMAB IN ANKYLOSING SPONDYLITIS PATIENTS FROM A PROSPECTIVE OBSERVATIONAL REGISTRY}

Proton Rahman ${ }^{1}$, Derek Haaland ${ }^{2}$, Dalton Sholter ${ }^{3}$, Michael Starr ${ }^{4}$, Arthur Karasik ${ }^{5}$, Michelle Teo ${ }^{6}$, Sanjay Dixit ${ }^{7}$, Ariel Masetto ${ }^{8}$, Anna Jarosynska ${ }^{9}$, Pauline Boulos ${ }^{7}$, Maqbool Sheriff ${ }^{10}$, Jacqueline Stewart ${ }^{11}$, Emmanouil Rampakakis ${ }^{12}$, Odalis Asin Miilan ${ }^{13}$, Allen Lehman ${ }^{13}$, Meagan Rachich ${ }^{14}$, Francois Nantel $\left.\right|^{13} .{ }^{1}$ Memorial University of Newfoundland, St. John's, Canada; ${ }^{2}$ The Waterside Clinic, Barrie, Canada; ${ }^{3}$ University of Alberta, Edmonton, Canada; ${ }^{4}$ McGill University Health Centre, Montréal, Canada; ${ }^{5}$ Ontario Rheumatology Association, Aurora, Canada; ${ }^{6}$ Balfour Medical Clinic, Penticton, Canada; ${ }^{7}$ McMaster University, Hamilton, Canada; ${ }^{8}$ Université de Sherbrooke, Sherbrooke, Canada; ${ }^{9}$ Private Clinic, Burlington, Canada: ${ }^{10}$ Nanaimo Regional General Hospital, Nanaimo, Canada: ${ }^{11}$ Penticton Regional Hospital, Penticton, Canada; ${ }^{12}$ JSS Medical Research, Montreal, Canada; ${ }^{13}$ Janssen Inc, Toronto, Canada; ${ }^{14}$ Janssen Inc., Toronto, Canada

Background: Long-term registries are essential to evaluate new therapies in a patient population that differs from clinical trial and usually varies over time.

Objectives: To describe the profile of ankylosing spondylitis (AS) patients treated with infliximab (IFX) or golimumab (GLM) treatment in Canadian routine care along with its long-term effectiveness and safety.

Methods: 810 AS patients treated with IFX or GLM were enrolled into the Biologic Treatment Registry Across Canada (BioTRAC) registry between 2005-2015 and 2010-2017, respectively. Study visits occurred at baseline and every 6 months thereafter, as needed per routine care. Effectiveness was assessed with changes in ASDAS, BASDAI, BASFI, MDGA, HAQ-DI, PtGA, back pain and acute phase reactants. Safety was evaluated with the incidence of adverse events (AEs) and drug survival rates.

Results: Of the 389 IFX- and 421 GLM-treated patients, the proportion of males were $62.7 \%$ and $59.1 \%$, the mean age were 45.6 and 45.7 years and the mean disease duration were 8.6 and 6.0 years, respectively. Most patients were bio-naive $(>82.7 \%)$. Interestingly, we observed a significant decrease in disease duration in the IFX cohort from a median of 8.0 to 3.5 and 1.0 years in 2005-2008, 2009-2012 and 2013-2015, respectively $(p<0.001)$. A reduction in baseline BASFI score $(6.3$ vs. 5.9 vs $5.1 ; P=0.011)$ and in the proportion of patient in ASDAS very high disease activity $(48.4 \%, 43.8 \%, 30.3 \% ; \mathrm{p}=0.004)$ were also observed. As for the GLM cohort, most disease parameters including median disease duration (1.6 years), mean baseline BASFI (5.3) and the proportion of patients in ASDAS very high disease activity $(48 \%)$ remained similar from 2010-2017.

Treatment with both IFX and GLM significantly improved all disease parameters over time $(P<0.001)$ from baseline up to 120 and 84 months, respectively, with similar efficacy between agents.

AEs were reported for $67.9 \%$ and $70.5 \%$ (136 and 131 events/100 PYs) and SAEs for $15.4 \%$ and $8.1 \%$ (10.5 and 22.7 events/100 PYs) covering 1251.3 and 674.8 years of exposure for IFX- and GLM-treated patients, respectively. The most frequently occurring $\mathrm{AEs}$ ( $>7 \%$ of patient in either group) were upper respiratory tract infection, arthralgia and back pain. Two deaths occurred in IFX-treated patients (myocardial infarct, drowning) and two among GLM-treated patients (oropharyngeal cancer; neutropenia, staphylococcal/pseudomonas infections, septic shock).

The proportion of patients who discontinued treatment were $65.8 \%$ over a mean 3.2 years of exposure in the IFX cohort and $56.8 \%$ over 1.6 years in the GLM cohort

Conclusion: Both IFX and GLM treatment significantly reduced disease activity and improved functionality in a similar fashion and were well tolerated in patients with AS. Differences in baseline characteristics over time demonstrate improvement in early diagnosis of AS and earlier access to biologic therapies.
Disclosure of Interests: Proton Rahman: None declared, Derek Haaland Grant/research support from: Janssen Sponsored Study, Dalton Sholter Grant/research support from: Janssen Sponsored Study, Michael Starr: None declared, Arthur Karasik: None declared, Michelle Teo Grant research support from: Janssen Sponsored Study, Sanjay Dixit Grant/ research support from: Janssen Sponsored Study, Consultant for: Janssen, Speakers bureau: Janssen, Ariel Masetto Grant/research support from: Amgen, Sanofi, Consultant for: Sanofi, Pfizer, Bristol-Myers Squibb, Novartis, Boehringer Ingelheim, Speakers bureau: Novartis, Anna Jarosynska Grant/research support from: Janssen Sponsored Study, Pauline Boulos Grant/research support from: Janssen Sponsored Study, Maqbool Sheriff Grant/research support from: Janssen Sponsored Study, Jacqueline Stewart Consultant for: Pfizer, Abbvie, Amgen, Celgene, Roche, Novartis, Merck, Emmanouil Rampakakis : None declared, Odalis Asin Mlilan Employee of: Employee of Janssen, Allen Lehman Employee of: Employee of Janssen, Meagan Rachich Shareholder of: Janssen, Employee of: Employee of Janssen, Francois Nantel Shareholder of: Janssen, Employee of: Employee of Janssen

DOI: 10.1136/annrheumdis-2019-eular.1431

\section{AB0705 AQUILA STUDY IN GERMANY - REAL WORLD ADHERENCE AND PERSISTENCE OF SECUKINUMAB TREATMENT IN ANKYLOSING SPONDYLITIS AND PSORIATIC ARTHRITIS PATIENTS - AN INTERIM ANALYSIS}

Uta Kiltz ${ }^{1}$, Daniel Peterlik ${ }^{2}$, Veronika Winkelmann ${ }^{2}$, Hans-Peter Tony ${ }^{3}$, on behalf of the AQUILA Study Group. ${ }^{1}$ Rheumazentrum Ruhrgebiet, Herne, and Ruhr University, Bochum, Germany; ${ }^{2}$ Novartis Pharma GmbH, Clinical Research Immunology, Hepatology and Dermatology, Nürnberg, Germany; ${ }^{3}$ Medizinische Klinik II, Universitätsklinik, Rheumatology/Immunology, Würzburg, Germany

Background: Secukinumab (SEC) has been shown to be an effective treatment for patients (pts) with ankylosing spondylitis (AS) and psoriatic arthritis (PsA) in several phase III studies ${ }^{1,2}$. Clinical studies reduce confounding factors, but do not guarantee the same results in real world where treating physicians deal with comorbidities, adherence and persistence challenges. Especially medication adherence has a direct impact on health outcomes. However, there is limited information on the adherence and persistence of SEC in routine care.

Objectives: The aim of this interim analysis is to report baseline (BL) characteristics and to assess adherence rate as well as treatment persis tence of SEC in AS and PSA pts under real world conditions.

Methods: AQUILA is an ongoing, non-interventional study enrolling 2000 pts with active AS or PsA in Germany. Adherence was calculated as the proportion of the accumulated actual dose divided by the accumulated target dose (according to summary of product characteristics) during the maintenance phase (week 6 to 52). Adherence rate was calculated as the proportion of pts who achieved an adherence of $>80 \%$ at wk 52 These calculations included pts who discontinued treatment with SEC; still ongoing pts were excluded from this interim analysis. Treatment persistence (time from study inclusion, i.e. date of informed consent, until treatment discontinuation) stratified by biologic/biosimilar (Bx) pretreatment was analyzed using Kaplan-Meier plots.

Results: This interim analysis describes 952 pts (AS $n=311$, PsA $n=641$ ) who were included at BL. In total, $51.4 \%(n=489)$ of the pts were female and $48.6 \%(n=463)$ male, mean age was 50.8 years, and $67.8 \%(n=645)$ were pretreated with Bx. At treatment start with SEC, pts presented with a number of extra-articular manifestations/comorbidities: plaque psoriasis $11.6 \%(n=36)$ in AS and $66.3 \%(n=425)$ in PsA, uveitis $6.4 \%(n=20)$ in AS and $1.7 \%(n=11)$ in PsA, depression $15.4 \% \quad(n=48)$ in AS and $15.4 \%$ $(n=99)$ in PsA, coronary heart disease $3.5 \%(n=11)$ in AS and $7.9 \%$ $(n=51)$ in PsA, stroke $0.6 \%(n=2)$ in $A S$ and $1.9 \% \quad(n=12)$ in PsA, and heart insufficiency $1.6 \%(n=5)$ in AS and $3.0 \%(n=19)$ in PsA.

Adherence rates for SEC $[95 \% \mathrm{Cl}]$ at week 52 were $64.5 \%[58.3,70.5$ and $56.0 \%[51.5 ; 60.4]$ for AS and PsA, respectively.

In both $\mathrm{AS}$ and $\mathrm{PsA}$, more Bx-pretreated pts discontinued treatment than Bx-naïve pts (AS: $38.0 \%$ versus $18.9 \%$; PsA: $32.3 \%$ versus $20.7 \%$ ). The most frequent reason for discontinuation was "adverse event/insufficient response/loss of efficacy" in both Bx-pretreated ( $n=73, \quad 33.0 \% / n=111$, $26.2 \%)$ as well as in Bx-naïve $(n=10,11.1 \% / n=34,15.7 \%)$ AS/PsA pts. Moreover, Kaplan-Meier analysis showed that in both AS (Figure 1a) and PsA (Figure 1b) groups, Bx-naïve pts who received SEC as first line therapy had a higher persistence rate than those pretreated with Bx. 
a

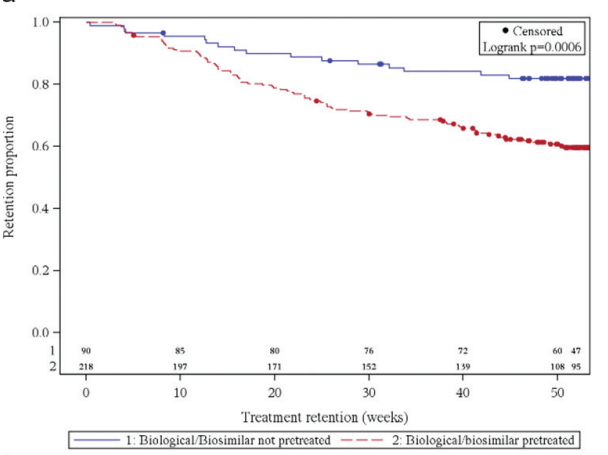

b

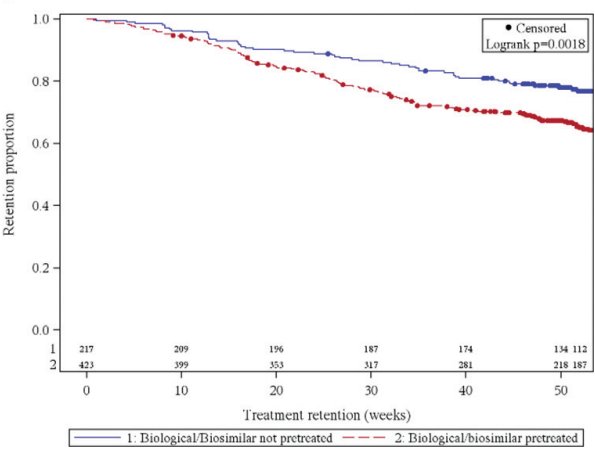

Figure 1.

Conclusion: AQUILA study revealed high disease burden at BL across AS and PSA pts characterized by the presence of extra-articular manifestations and cardiovascular diseases. Adherence rates under real-world conditions were consistent and comparable with published literature regarding immune-mediated inflammatory diseases ${ }^{3}$. SEC demonstrated a high drug persistence in real world in particular in Bx-naive pts. Overall, this interim analysis demonstrated that SEC is a reliable treatment in Bxnaïve as well as Bx-pretreated pts with $A S$ or PSA in a daily routine setting.

\section{REFERENCES}

[1] Baeten D et al. Lancet. 2013;382(9906):1705-13;

[2] Mclnnes IB et al. Lancet. 2015;386(9999):1137-46;

[3] Vangeli E et al. Adv Ther. 2015;32:983-1028

Disclosure of Interests: Uta Kiltz Grant/research support from: AbbVie, Chugai, Eli Lilly, Grünenthal, Janssen, MSD, Novartis, Pfizer, Roche, and UCB., Consultant for: AbbVie, Chugai, Eli Lilly, Grünenthal, Janssen, MSD, Novartis, Pfizer, Roche, and UCB. Daniel Peterlik Employee of: Novartis Pharma $\mathrm{GmbH}$, Veronika Winkelmann Employee of: Novartis Pharma GmbH, Hans-Peter Tony Consultant for: Eli Lilly and Company, Speakers bureau: Eli Lilly and Company

DOI: 10.1136/annrheumdis-2019-eular.435

\section{\begin{tabular}{|l|l}
\hline AB0706 DIFFERENCES IN RHEUMATOLOGISTS' TREATMENT & \\
\hline
\end{tabular} PREFERENCES FOR ANKYLOSING SPONDYLITIS AND NON- RADIOGRAPHIC AXIAL SPONDYLOARTHRITIS}

Phil pouliot, Lynn Price. Spherix Global Insights, Exton, United States of America

Background: The lack of a formal indication for Non-Radiographic Axial Spondyloarthritis ( $\mathrm{nr}-\mathrm{axSpA}$ ) has meant that many US rheumatologists fundamentally treat it as an early manifestation of Ankylosing Spondylitis (AS) and seldom differentiate their treatment approach when using biologics/small molecules.

Objectives: This study sought to gain a better understanding of the number of AS and nr-axSpA patients rheumatologists manage, recent changes made in treatment, and how AS and nr-axSpa patients are differentiated beyond radiographic evidence. The study also evaluated the differences in use of biologics/small molecules for the treatment of AS and $\mathrm{nr}-\mathrm{axSpA}$ and rheumatologist projected changes in the management of these two conditions.
Methods: An independent market analytics firm administered a survey with US rheumatologists $(n=104)$ on the treatment landscape for AS and $n r-a x S p A$. To qualify, rheumatologists had to spend the majority of their professional time in clinical practice, manage a minimum of $50 \mathrm{AS}$ or $\mathrm{nr}$ axSpA patients, and have been in practice between 2 and 35 years Respondents answered questions pertaining to their practice, the demographics of their AS/nr-axSpA patients, their treatment approaches for each type, as well as attitudinal statements. The survey was administered online in February 2018 and respondents were compensated for their participation. A similar study was also conducted in 2017 allowing for year over year comparison. The data were collected and analyzed in SPSS

Results: Three-quarters of rheumatologists reported they treated their AS and nr-axSpA patients in a similar manner, though there was a higher use of NSAIDs and local steroids in non-radiographic disease, and more biologic/JAK use in AS. More established TNF inhibitors such as adalimu mab, etanercept, and infliximab dominated biologics/small molecule preference, collectively accounting for approximately three-quarters of al biologic-treated AS and nr-axSpA patients. However, use of adalimumab, etanercept, and infliximab was expected to decline or stay flat in the next three months, while use of secukinumab, tofacitinib, golimumab IV and both infliximab biosimilars, was anticipated to increase.

Although not indicated for either condition, $28 \%$ of respondents reported off-label use of tofacitinib for AS, and $25 \%$ for nr-axSpA, a significant increase from 2017. Projected off-label use of tofacitinib in the following three months is also expected to increase.

Conclusion: Rheumatologists are less likely to use biologics/small molecules for nr-axSpA, but when they do, use of brands is similar to those used in AS. While TNF inhibitors continue to account for the majority of biologic/small molecule prescriptions for these conditions, rheumatologists project increased use of agents with alternative mechanisms of action in the future.

Disclosure of Interests: None declared

DOI: 10.1136/annrheumdis-2019-eular.7752

\section{AB0707 LTBI SCREENING IN SPONDYLOARTHRITIS PATIENTSPRIOR TO ANTI-TNF TREATMENT AND FOLLOW-UP IN AN ENDEMIC AREA}

Andrea Shimabuco, Ana Medeiros, Renata Miossi, Karina Bonfiglioli, Julio Moraes, Celio Gonçalves, Percival D. Sampaio-Barros, Claudia Goldenstein-Schainberg, Fernando Souza, Leandro Prado, Michelle Lopes, Eloisa Bonfa, Carla Saad. Hospital das Clinicas da Faculdade de Medicina da Universidade de São Paulo, Rheumatology Division, Sao Paulo, Brazil

Background: Screening for latent tuberculosis infection (LTBI) remains a concern in endemic regions for anti-TNF eligible patients and recent researches have shown peculiarities between the inflammatory arthropathies such as different groups of spondyloarthritis ( $\mathrm{SpA})$.

Objectives: To evaluate the long-term efficacy of LTBI screening and treatment in patients with Ankylosing Spondylitis (AS) and Psoriatic Arthritis $(P s A)$ receiving TNF blockers in a single center.

Methods: A total of $218 \mathrm{SpA}$ patients (135 AS and 83 APS) were screened for LTBI before receiving anti-TNF (infliximab, adalimumab, etanercept, certolizumab pegol and golimumab) treatment using the tuberculin skin test (TST), chest X-ray (CXR) and history of previous exposure to tuberculosis (TB). Patients were regularly followed every 2-3 months and asked about infectious symptoms or new exposure. TST was not repeated regularly. $\mathrm{LTBI}$ patients were treated with isoniazid $(300 \mathrm{mg} / \mathrm{day})$ for 6 months, according local guidelines.

Results: One hundred and eight patients (49.5\%) were treated with a single anti-TNF agent and the total duration of biological treatment was approximately six years $(5.9 \pm 4.0)$. INF and ADA were most often used among the 422 treatment cycles analyzed, representing $45 \%$ and $30 \%$ respectively. LTBI screening was positive in 82 patients (38\%): 69 (84\%) were TST-positive, $23(28 \%)$ had a history of TB exposure and $5(6 \%)$ had an abnormal CXR. As isolated variables TST positivity and previous exposure accounted for 58(71\%) and 11 (13\%) LTBI diagnosis. There were some distinct patterns between APS and AS patients screening: despite APS patients had more cases of previous TB than AS patients $(6 \%$ vs $0.7 \%, P=0.03)$, they had a lower frequency of LTBI $(30 \%$ vs $42 \%, P=0.04)$. Among LTBI patients, TST positivity was lower in SpA than AS patients $(64 \%$ vs $93 \%, P=0.002)$, even with more previous exposure $(52 \%$ vs $18 \%, P=0.02)$ and in patients with peripheral arthritis ( $27 \%$ vs $42 \%, P=0.03$ ). During follow-up, 11 patients developed active TB: 5 under ADA, 5 under INF and 1 under ETA treatment. Five cases (45\%) were extrapulmonary: 3 pleural, 1 peritoneal and 1 spondylodiscitis. Four (36\%) cases occurred in patients with a positive LTBI screening and 7 in patients without LTBI. There was no difference in drug survival 